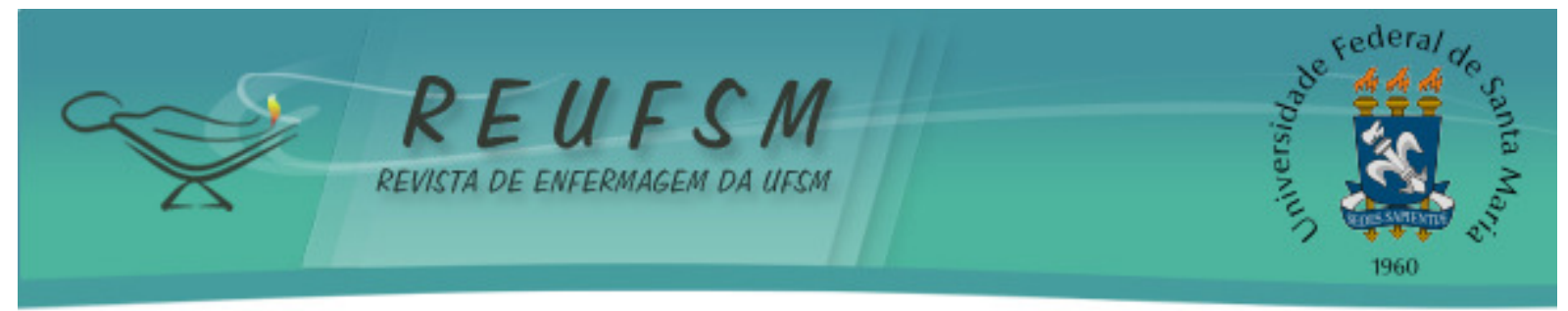

ARTIGO ORIGINAL

\author{
ASSOCIAÇÃO ENTRE ANTECEDENTES GINECOLÓGICO-OBSTÉTRICOS E SINTOMAS DO \\ CLIMATÉRIO \\ ASSOCIATION BETWEEN RECORD GYNECOLOGICAL-OBSTETRICS AND CLIMACTERIC \\ SYMPTOMS
}

\title{
ASSOCIAÇÃO ENTRE ANTECEDENTES GINECOLÓGICO-OBSTÉTRICOS E SINTOMAS DO CLIMATÉRIO
}

Doi: $10.5902 / 2179769210567$

Estela Rodrigues Paiva Alves ${ }^{1}$ Juliana Cristina Cruz Calazans ${ }^{2}$ Almiranice Yara de Moura Ferreira ${ }^{3}$ Gerlaine de Oliveira Leite ${ }^{4}$ Khivia Kiss da Silva Barboza ${ }^{5}$ Maria Djair Dias ${ }^{6}$

RESUMO: Objetivo: verificar a associação entre os antecedentes ginecológico-obstétricos e sintomas do climatério. Método: pesquisa exploratório-descritiva, transversal com abordagem quantitativa, envolvendo 260 mulheres no climatério. Foram excluídas as mulheres que faziam tratamento de reposição hormonal, uso de fitoestrógenos e contraceptivos hormonais até seis meses antes da coleta. Utilizou-se para a coleta dos dados dois questionários, um com dados ginecológicos e obstétricos e o Índice Menopausal de Blatt \& Kupperman. Os dados foram analisados por meio das técnicas de estatística descritiva e inferencial. Resultados: os sintomas de grau leve foram os mais apontados pelas mulheres, os mesmos estiveram associados aquelas que referiram ainda apresentar fluxo menstrual regular. Conclusão: o histórico ginecológico-obstétrico pode ter relação com os possíveis sintomas do climatério.

Descritores: Climatério; Saúde da Mulher; Enfermagem.

ABSTRACT: Aim: to check the association between the gynecological-obstetric background and menopausal symptoms. Method: exploratory research, descriptive, cross-sectional of quantitative approach, involving 260 climateric women. We excluded women who were hormone replacement therapy, use hormonal contraceptives and phytoestrogens up to six months prior to collection. Was used for data collection two questionnaires, one with gynecological-obstetric data and the Blatt\&Kupperman Menopausal Index. Data were analyzed using the techniques of descriptive and inferential statistics. Results: the mild symptoms were the most frequently reported by women, they were associated with those

\footnotetext{
${ }^{1}$ Enfermeira. Doutoranda em Enfermagem pelo Programa de Pós-Graduação em Enfermagem da Universidade Federal da Paraíba (PPGEnf/UFPB). Membro do Grupo de Estudos e Pesquisa em História Oral e Saúde da Mulher - GEPHOSM da UFPB. João Pessoa (PB), Brasil. E-mail: rodrigues.estela@gmail.com

${ }^{2}$ Graduanda em Enfermagem. Universidade Federal de Pernambuco (UFPE). Recife (PE), Brasil. E-mail: juliana.calazans@ufpe.br

${ }^{3}$ Graduanda em Enfermagem. Universidade Federal de Pernambuco (UFPE). Recife (PE), Brasil. E-mail: yara_nicinha@yahoo.com.br

${ }^{4}$ Graduanda em Enfermagem. Universidade Federal de Pernambuco (UFPE). Recife (PE), Brasil. E-mail: gerlaine.oliveira@gmail.com

${ }^{5}$ Enfermeira. Docente do Curso de Enfermagem da Universidade Federal de Campina Grande (UFCG). Campina Grande (PB), Brasil. E-mail: khiviakiss@yahoo.com.br

${ }^{6}$ Enfermeira. Docente do Programa de Pós-Graduação em Enfermagem da Universidade Federal da Paraíba (PPGEnf/UFPB). Membro do Grupo de Estudos e Pesquisa em História Oral e Saúde da Mulher - GEPHOSM da UFPB. João Pessoa (PB), Brasil. E-mail: mariadjair@yahoo.com.br
} 


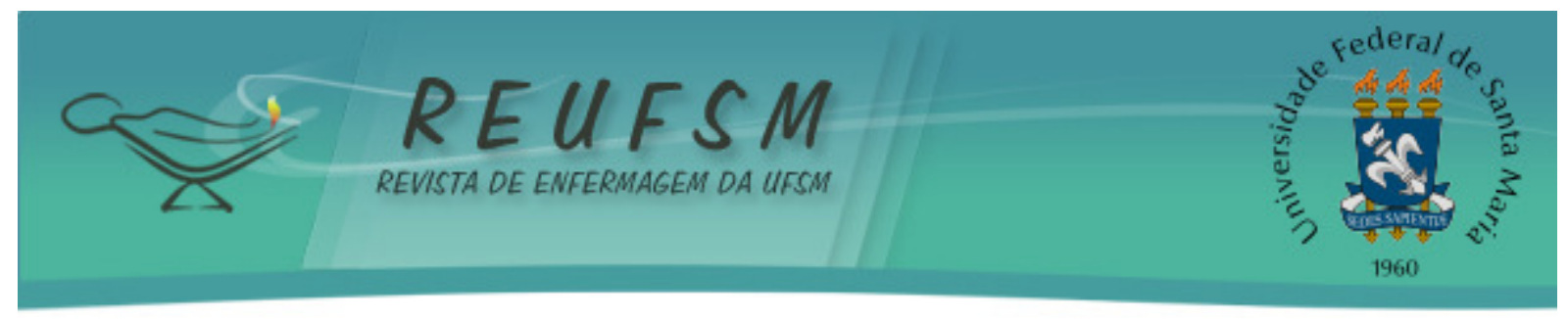

who also reported regular menstrual flow. Conclusion: the gynecological-obstetrical history may be related to the possible symptoms of menopause.

Descriptors: Climacteric; Women's Health; Nursing.

RESUMEN: Objetivo: verificar la asociación entre los antecedentes ginecológicosobstétricos y síntomas del climaterio. Método: investigación exploratoria-descriptiva, con enfoque cuantitativo, con la participación de 260 mujeres en el climaterio. Fueron excluidas las mujeres que hacían uso de la terapia de reemplazo de hormonas, fitoestrógenos y contraceptivos hormonales hasta seis meses antes de la colección. Fueron utilizados para los datos de la colecta dos cuestionarios, uno con datos ginecológicos y obstétricos y otro con el índice Menopausal Index Blatt \& Kupperman. Los datos fueron analizados por medio de las técnicas estadística descriptiva $e$ inferencial. Resultados: los síntomas leves fueron los más frecuentemente reportados por las mujeres, que también se asociaron con las que presentaron flujo menstrual regular. Conclusión: la historia ginecológica-obstétrica puede estar relacionada con los posibles síntomas del climaterio.

Descriptores: Climaterio; Salud de la Mujer; Enfermería.

\section{INTRODUÇÃO}

O climatério é marcado pela transição do estágio reprodutivo para o não reprodutivo no processo de vida natural da mulher. É caracterizado por uma série de eventos endócrinos que podem levar a queixas e sintomas que variam na sua intensidade e diversidade em consequência das alterações ovarianas com gradativa redução da produção estrogênica marcado pelo estado de hipogonadismo hipergonadotrófico. ${ }^{1}$

No período inicial do climatério as alterações hormonais são mais intensas. Com a diminuição da resposta ovariana aos hormônios gonadotróficos, os folículos diminuem a produção de estradiol. É neste ciclo vital da mulher que o corpo lúteo também já não produz progesterona suficiente para suprir as suas necessidades fisiológicas. ${ }^{1}$

A fase climatérica situa-se na faixa etária dos 40 a 65 anos de idade e se divide em três períodos sequenciais - pré-menopausa, perimenopausa e pós-menopausa, cada um com características próprias que variam de acordo com o padrão menstrual e fertilidade da mulher durante a sua vida reprodutiva. ${ }^{1}$

A Sociedade Internacional da Menopausa ${ }^{2}$ afirma que este período de transição da vida da mulher nem sempre está associado à sintomatologia, porém com o aparecimento dos sintomas, pode ser definida como "síndrome do climatério". Esta síndrome pode envolver modificações de ordem física, emocional e social na vida da mulher. ${ }^{3}$

A definição de pré-menopausa é usada de forma ambígua, podendo se referir a um ou dois anos, anterior a instalação da menopausa ou ao tempo total do período reprodutivo, ou seja, desde a menarca até a instalação da menopausa. Diante dessa ambiguidade, recomenda que este termo (pré-menopausa) seja utilizado para designar o final do período reprodutivo, caracterizado pelo último período menstrual, que só poderá ser reconhecido após 12 meses do último ciclo menstrual e sugere que este, seja substituído pelo termo "transição para a menopausa". ${ }^{4}$

Já outra fase, conhecida como perimenopausa abrange o período imediato anterior à cessação da menstruação (menopausa) até o primeiro ano após a instalação (12 meses sem ciclos menstruais) da menopausa, somente reconhecida retrospectivamente após 12 meses de amenorreia, sem qualquer causa patológica ou fisiológica óbvia, ocorrendo em média por volta dos 50 anos de idade, em mulheres brasileiras. ${ }^{1-2}$ 


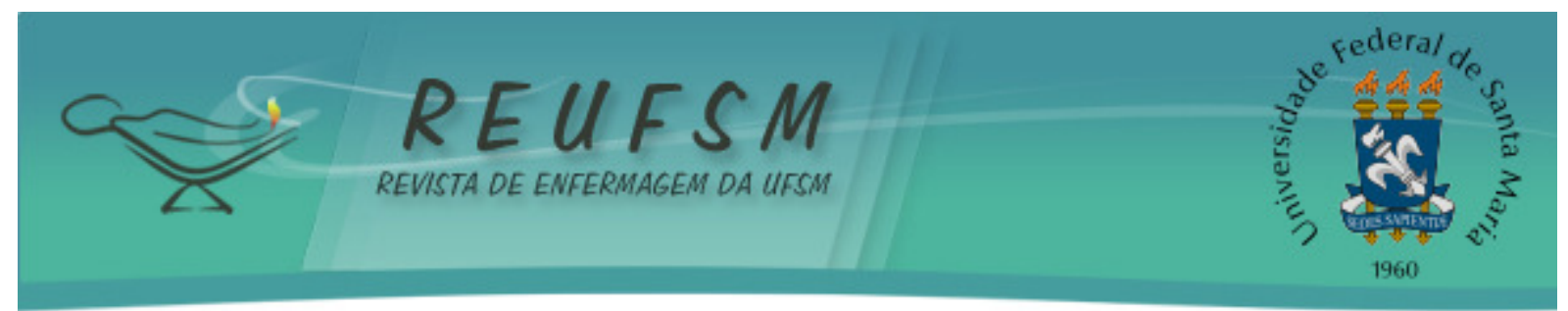

Fatores como baixo nível socioeconômico, tabagismo e peso baixo podem antecipar a menopausa. Já os fatores que reduzem os ciclos ovulatórios, como o uso de contraceptivos e paridade, pode adiar este período. ${ }^{4}$

Mulheres histerectomizadas apresentam menopausa artificial, muito embora o funcionamento dos ovários esteja preservado. Porém, na ooforectomia bilateral (retirada dos ovários), a sintomatologia pode apresentar-se mais agressivamente com um aumento da frequência e intensidade dos sintomas climatéricos. ${ }^{1}$

A menopausa induzida ocorre quando a mulher cessa seu ciclo menstrual em decorrência da remoção cirúrgica de ambos os ovários com ou sem histerectomia ou ablação iatrogênica das funções ovarianas como pode ocorrer nos casos de quimioterapia, radioterapia e menopausa precoce ou prematura, quando esta ocorre em idade inferior a 40 anos. $^{2}$

$\mathrm{E}$, finalmente a pós-menopausa, é caracterizada como o período após a instalação da menopausa ou parada da menstruação, seja ela induzida ou natural, se estendendo até os 65 anos de idade. ${ }^{1-2}$

As alterações fisiológicas do climatério no ciclo vital de uma mulher, mesmo com sintomas de intensidades diferentes, geram consequências que podem afetar o seu bem-estar físico e mental. ${ }^{5}$

A implantação da atenção à saúde da mulher no climatério pressupõe a existência de profissionais de saúde capacitados para atender as particularidades dessa clientela. No entanto, na prática, nos deparamos com uma assistência fragmentada na qual a mulher não recebe a atenção adequada, muitas vezes, devido a falta de preparação dos profissionais de saúde que deixam passar despercebidas algumas das características importantes e sinalizadoras da fase do climatério que podem indicar possíveis alterações na saúde da mulher.

Sendo assim, é imprescindível abordar a temática do climatério junto aos profissionais de saúde e dando ênfase aos profissionais de enfermagem, uma vez que os enfermeiros são profissionais que se destacam na educação em saúde, sendo esta uma das intervenções necessárias para orientar as mulheres sobre essa nova etapa de suas vidas, além desta se tratar de um dispositivo essencial para a promoção da saúde e a prevenção de doenças. ${ }^{6}$

Conhecer a relação entre as condições ginecológicas e obstétricas sobre o período do climatério, incluindo a instalação da menopausa e a intensidade dos sintomas se faz pertinente no sentido de contribuir para a manutenção de uma assistência de enfermagem mais integral e humanizada. Com base nos argumentos acima se buscou esclarecer o seguinte questionamento: existe associação entre o histórico ginecológico-obstétrico e os possíveis sintomas do período do climatério entre mulheres no climatério? Assim, o presente estudo objetivou verificar a associação entre os antecedentes ginecológico-obstétricos e sintomas do climatério.

\section{MÉTODO}

Trata-se de uma pesquisa exploratório-descritiva, transversal com abordagem quantitativa, envolvendo mulheres no climatério. Foi conduzido no Programa Academia da Cidade (PAC), implantado pela Secretaria de Saúde da Cidade do Recife em 2002, como uma Política de Promoção à Saúde, inserida no Sistema Único de Saúde (SUS), focado no oferecimento de atividade física, alimentação saudável e lazer, tendo como principal objetivo melhorar a qualidade de vida da população recifense. ${ }^{7}$

A seleção da amostra se deu por acessibilidade devido ao fato de não haver um cadastro dos usuários. Devido a esta limitação, não foi possível definir o tamanho da amostra. Sendo assim, optou-se por abordar todas as mulheres que frequentaram os pólos do PAC, nos meses de julho, agosto e setembro de 2011, período da coleta de dados.

Neste período foram abordadas 260 mulheres que atenderam os critérios de inclusão e aceitaram participar da pesquisa livremente confirmando sua participação por meio da assinatura do Termo de Consentimento Livre e Esclarecido. Assim, os critérios de inclusão foram: além da anuência da entrevistada, ter entre 40 e 65 anos de idade ${ }^{1}$ e frequentar o PAC 


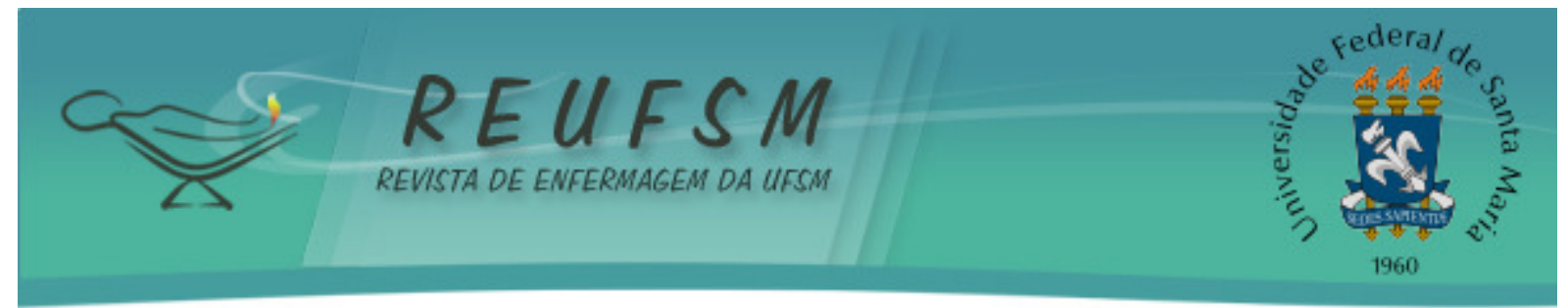

a pelo menos seis meses. Optou-se por excluir as mulheres que faziam tratamento de reposição hormonal, incluindo o uso de fitoestrógenos e contraceptivos hormonais até seis meses antes da sua possível inclusão devido a estas substâncias interferirem na sintomatologia do climatério. ${ }^{8}$

Para coleta dos dados foi utilizado um questionário, contendo dados socioeconômico e demográfico; antecedentes ginecológicos e obstétricos e o Índice Menopausal de Blatt \& Kupperman (IMBK). ${ }^{9}$ Os sintomas do climatério foram identificados por meio do IMBK. Trata-se de um instrumento já validado no Brasil e utilizado para especificar e classificar os sinais e sintomas do climatério. Envolve 11 sintomas ou queixas (sintomas vasomotores, parestesia, insônia, nervosismo, melancolia, vertigem, fadiga, artralgia/mialgia, cefaleia, palpitações e formigamento). ${ }^{9}$

O IMBK permite classificar os sintomas, segundo a intensidade referida pela entrevistada. Para cada sintoma é estabelecido um peso (escore) diferente que varia de leve (resultado menor ou igual a 19); moderado (se for entre 20 e 25) e intenso (se maior que 35), neste último, uma pontuação maior que 35 sugere maior aproximação da menopausa, período caracterizado pela ausência da menstruação confirmada após 12 meses. Para obter o total de pontos, basta somar os escores e quanto maior for a pontuação obtida, mais intensa será a sintomatologia da mulher. ${ }^{9}$

Os dados foram agrupados e analisados por meio do programa estatístico SPSS (Statistical Package for the Social Sciences) na versão 15. Utilizou-se para análise as técnicas de estatística descritiva (distribuições absolutas, percentuais uni e bivariadas e as medidas: média, mediana e desvio padrão) e inferencial por meio do teste Exato de Fisher desde que as condições para utilização do teste qui-quadrado não foram verificadas. O nível de significância utilizado nas decisões dos testes foi de $5 \%$.

A pesquisa foi submetida à apreciação do Comitê de Ética e Pesquisa da Universidade de Pernambuco, atendendo às orientações que rege a Resolução 196/96 do Conselho Nacional de Saúde ${ }^{10}$ do qual obteve parecer favorável, com protocolo $n^{\circ}$ 059/11.

\section{RESULTADOS E DISCUSSÃO}

\section{Caracterização da população}

A idade média do total de entrevistadas $(n=260)$ foi de $51(+/-7,22)$ anos com uma mediana de 50 anos. Quase metade das mulheres $(47,7 \%)$ tinha entre 40 e 49 anos. Dois terços $(66,2 \%)$ se declarou como não branca, sendo que a maioria $(82,3 \%)$ vivia em união estável. Em relação à religião, a católica foi a de maior percentual (72,7\%). Quanto à escolaridade, 58,5\% estudou oito ou mais anos. Em relação à profissão/ocupação, um pouco mais da metade $(63,5 \%)$ exercia atividade remunerada, sendo o restante das mulheres $(36,5 \%)$, donas de casa e quase metade das mulheres tinha uma renda per capita maior que um salário mínimo $(44,2 \%)$, sendo estes valores calculados com base no salário mínimo vigente (R\$ 540,00) em 2011.

Além das modalidades de ginástica e caminhada, oferecidas pelo PAC, as mulheres também praticavam natação e hidroginástica, como pode ser visto na Tabela 1.

Tabela 1 - Distribuição das mulheres no climatério do Programa Academia da Cidade (PAC) de Recife/PE, jul-set, 2011, segundo a modalidade de atividade física exercida.

\begin{tabular}{lcc} 
Variável & $\mathrm{n}$ & $\%$ \\
\hline - Prática de atividade física & 7 & 2,7 \\
Natação & 46 & 17,7 \\
Hidroginástica & 211 & 81,2 \\
Caminhada & 229 & 88,1 \\
Ginástica & 260 & \\
BASE(1) &
\end{tabular}

(1): Considerando que uma mesma paciente tenha citado mais de uma resposta, considera a base para o cálculo dos percentuais e não o total.

Fonte: Associação entre antecedentes ginecológico-obstétricos e sintomas do climatério,2012. 


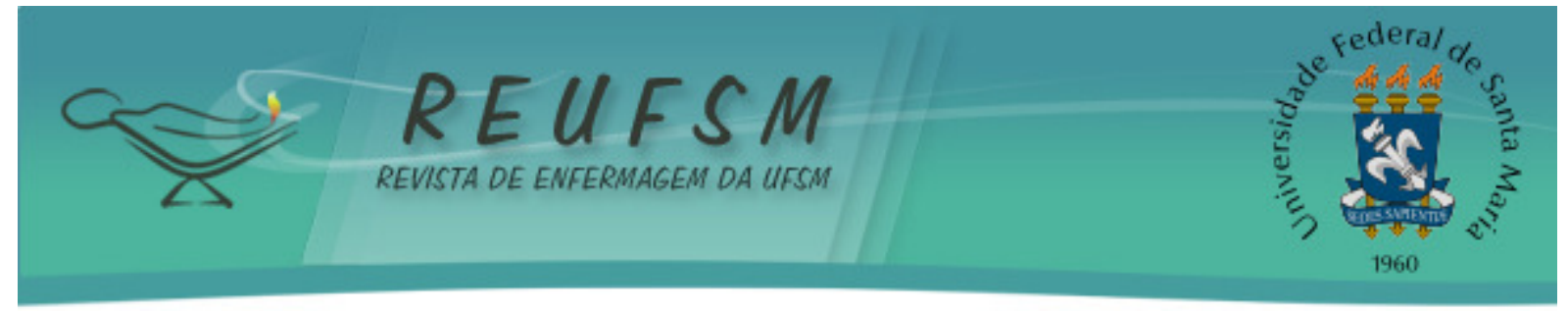

\section{Dados referentes aos objetivos propostos}

Em relação à intensidade dos sintomas, cerca da metade das mulheres apresentou manifestações leves e apenas um sexto relatava sintomatologia intensa como apresentado na Tabela 2.

Tabela 2 - Distribuição das mulheres no climatério do Programa Academia da Cidade (PAC) de Recife/PE, jul-set, 2011, segundo a classificação do IMBK.

\begin{tabular}{lcc}
\hline Variável & $\mathrm{N}$ & $\%$ \\
\hline - Escala de Blatt \& Kupperman & & \\
Manifestações leves & 196 & 75,4 \\
Manifestações moderadas & 52 & 20,0 \\
Manifestações intensas & 12 & 4,6 \\
\hline TOTAL & 260 & 100,0 \\
\hline
\end{tabular}

Fonte: Associação entre antecedentes ginecológico-obstétricos e sintomas do climatério, 2012.

Estudos têm utilizado o IMBK para identificação e classificação da intensidade dos sintomas no climatério. No Ambulatório de Ginecologia de uma Unidade Básica de Saúde, em São Paulo, foi realizado um estudo com 250 mulheres com idades acima de 45 anos utilizando o IMBK no qual foi evidenciado que a maioria das pesquisadas relatavam sintomatologia leve, entretanto estas faziam uso da terapia hormonal, já entre as que não faziam uso da terapia foram apresentados sintomas de intensidade moderada e acentuada. ${ }^{11}$ As mulheres avaliadas em nosso estudo, apesar de não fazerem uso da terapia de reposição hormonal, praticavam atividade física que parece influenciar na redução dos sintomas, conforme achados da literatura. ${ }^{12-13}$

Em mulheres que apresentam sintomas mais graves, a terapia de reposição hormonal é um tratamento importante, entretanto, seu uso em longo prazo pode acarretar vários efeitos colaterais como doenças cardiovasculares, osteoporose e câncer de mama. Devido a estes riscos o tratamento deve ser feito com o acompanhamento médico, em um período de no máximo cinco anos. ${ }^{14}$

No entanto, ao levar em consideração o período do climatério como uma fase da vida e não como um processo patológico, é fundamental que as mulheres sejam devidamente orientadas pelos profissionais de saúde sobre as possibilidades preventivas e terapêuticas, e, desse modo, não se tornem usuárias passivas de medicamentos. ${ }^{15}$

Vale salientar que no climatério a atividade física desempenha um papel fundamental, pois além de proporcionar bem estar, melhora na autoestima, nas funções motoras, resistência muscular, sociabilidade, diminuindo os riscos de doenças, melhorando a saúde psíquica e social, ela também proporciona uma melhora dos sintomas presentes nessa fase da vida da mulher (fogachos, irritabilidade, depressão, não aceitação desta fase, falta de libido, entre outros). ${ }^{3}$ Isso se dá, devido a adoção de um estilo de vida ativo contribuir para uma melhoria das condições de saúde e traz repercussões positivas tanto na esfera psíquica quanto nos sintomas característicos dessa fase. ${ }^{16}$

Referente às variáveis contidas na Tabela 3 foi verificada associação significativa da escala com cada uma das variáveis: presença de fluxo menstrual, idade da menopausa e fluxo regular e para as referidas variáveis, se destaca que, o percentual das mulheres classificadas no grau leve do IMBK foi mais elevado entre as que tinham fluxo menstrual $(84,2 \%)$. Os percentuais das mulheres com sintomas leves e moderados aumentaram com o avançar da idade (próximo ao início da menopausa), enquanto que o percentual com sintomas intensos reduziu nas mulheres acima dos 50 anos. Das 120 pesquisadas que tinham fluxo menstrual nenhuma teve sintomas intensos, o percentual com sintomas leves foi mais elevado entre as que tinham fluxo do que entre os que não tinham fluxo regular. 


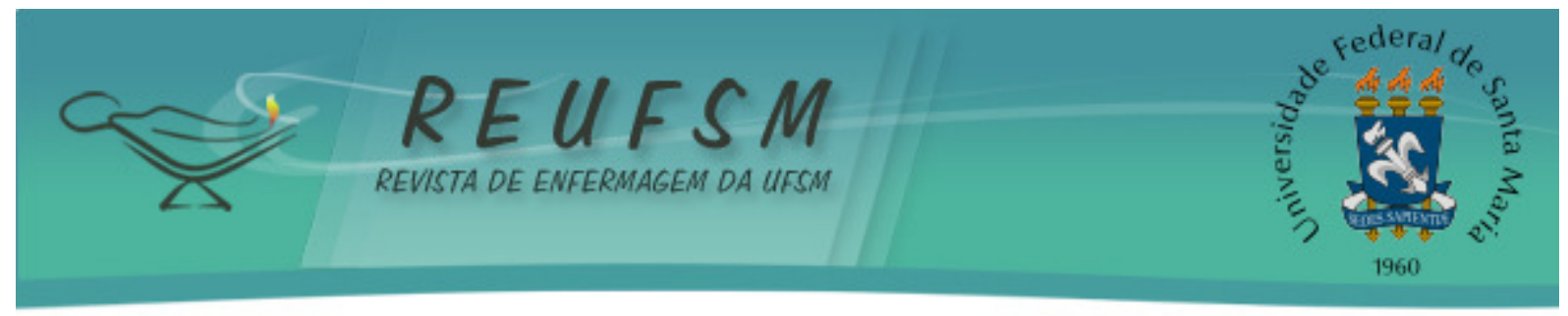

Tabela 3 - Avaliação dos sintomas de acordo com a escala do IMBK, segundo os antecedentes ginecológicos e obstétricos das mulheres no climatério do Programa Academia da Cidade (PAC) de Recife/PE, jul-set, 2011.

\begin{tabular}{|c|c|c|c|c|c|c|c|c|c|}
\hline \multirow{3}{*}{ Variável } & \multicolumn{6}{|c|}{ Escala de Blatt \& Kupperman } & \multirow{2}{*}{\multicolumn{2}{|c|}{ TOTAL }} & \multirow{3}{*}{ Valor de $\mathrm{p}$} \\
\hline & \multicolumn{2}{|c|}{ Leve } & \multicolumn{2}{|c|}{ Moderado } & \multicolumn{2}{|c|}{ Intenso } & & & \\
\hline & $\mathrm{N}$ & $\%$ & $\mathrm{~N}$ & $\%$ & $\mathrm{~N}$ & $\%$ & $\mathrm{~N}$ & $\%$ & \\
\hline \multicolumn{10}{|l|}{-Idade da menarca } \\
\hline Até 11 & 33 & 71,7 & 10 & 21,7 & 3 & 6,5 & 46 & 100,0 & $\mathrm{p}^{(1)}=0,931$ \\
\hline 12 a 14 & 126 & 75,9 & 33 & 19,9 & 7 & 4,2 & 166 & 100,0 & \\
\hline 15 ou mais & 37 & 77,1 & 9 & 18,8 & 2 & 4,2 & 48 & 100,0 & \\
\hline Grupo Total & 196 & 75,4 & 52 & 20,0 & 12 & 4,6 & 260 & 100,0 & \\
\hline \multicolumn{10}{|l|}{ - Idade sexarca } \\
\hline 13 a 15 & 17 & 63,0 & 10 & 37,0 & - & - & 27 & 100,0 & $\mathrm{p}^{(1)}=0,141$ \\
\hline 16 a 19 & 78 & 78,0 & 16 & 16,0 & 6 & 6,0 & 100 & 100,0 & \\
\hline 20 a 25 & 67 & 72,0 & 21 & 22,6 & 5 & 5,4 & 93 & 100,0 & \\
\hline 26 a 38 & 33 & 86,8 & 4 & 10,5 & 1 & 2,6 & 38 & 100,0 & \\
\hline Grupo Total & 195 & 75,6 & 51 & 19,8 & 12 & 4,7 & 258 & 100,0 & \\
\hline \multicolumn{10}{|l|}{-Paridade } \\
\hline Nenhum & 14 & 77,8 & 4 & 22,2 & - & - & 18 & 100,0 & $\mathrm{p}^{(1)}=0,881$ \\
\hline 1 a 2 & 112 & 74,2 & 30 & 19,9 & 9 & 6,0 & 151 & 100,0 & \\
\hline 3 ou mais & 70 & 76,9 & 18 & 19,8 & 3 & 3,3 & 91 & 100,0 & \\
\hline Grupo Total & 196 & 75,4 & 52 & 20,0 & 12 & 4,6 & 260 & 100,0 & \\
\hline \multicolumn{10}{|l|}{$\begin{array}{l}\text {-Presença de fluxo } \\
\text { menstrual }\end{array}$} \\
\hline Sim & 101 & 84,2 & 19 & 15,8 & - & - & 120 & 100,0 & $\mathrm{p}^{(1)}<0,001^{*}$ \\
\hline Cessou espontaneamente & 61 & 76,3 & 16 & 20,0 & 3 & 3,8 & 80 & 100,0 & \\
\hline Realizou histerectomia & 28 & 59,6 & 12 & 25,5 & 7 & 14,9 & 47 & 100,0 & \\
\hline Realizou ooferectomia & 6 & 46,2 & 5 & 38,5 & 2 & 15,4 & 13 & 100,0 & \\
\hline Grupo Total & 196 & 75,4 & 52 & 20,0 & 12 & 4,6 & 260 & 100,0 & \\
\hline \multicolumn{10}{|l|}{-Fluxo regular } \\
\hline Sim & 90 & 88,2 & 12 & 11,8 & - & - & 102 & 100,0 & $\mathrm{p}^{(1)}=0,009^{*}$ \\
\hline Não & 11 & 61,1 & 7 & 38,9 & - & - & 18 & 100,0 & \\
\hline Grupo Total & 101 & 84,2 & 19 & 15,8 & - & - & 120 & 100,0 & \\
\hline \multicolumn{10}{|l|}{$\begin{array}{l}\text {-Número de dias com } \\
\text { fluxo menstrual }\end{array}$} \\
\hline 1 a 3 & 50 & 84,7 & 9 & 15,3 & - & - & 59 & 100,0 & $\mathrm{p}^{(2)}=0,166$ \\
\hline 4 a 5 & 36 & 90,0 & 4 & 10,0 & - & - & 40 & 100,0 & \\
\hline 6 ou mais & 15 & 71,4 & 6 & 28,6 & - & - & 21 & 100,0 & \\
\hline Grupo Total & 101 & 84,2 & 19 & 15,8 & - & - & 120 & 100,0 & \\
\hline \multicolumn{10}{|l|}{-Idade da menopausa } \\
\hline 29 a 39 & 13 & 59,1 & 3 & 13,6 & 6 & 27,3 & 22 & 100,0 & $\mathrm{p}^{(1)}=0,014^{*}$ \\
\hline 40 a 49 & 48 & 67,6 & 17 & 23,9 & 6 & 8,5 & 71 & 100,0 & \\
\hline 50 a 59 & 29 & 72,5 & 11 & 27,5 & - & - & 40 & 100,0 & \\
\hline Grupo Total & 90 & 67,7 & 31 & 23,3 & 12 & 9,0 & 133 & 100,0 & \\
\hline \multicolumn{10}{|l|}{$\begin{array}{l}\text {-Tempo de menopausa } \\
\text { natural }\end{array}$} \\
\hline$<5$ anos & 23 & 69,7 & 7 & 21,2 & 3 & 9,1 & 33 & 100,0 & $\mathrm{p}^{(1)}=0,128$ \\
\hline$\geq 5$ anos & 38 & 80,9 & 9 & 19,1 & - & , & 47 & 100,0 & \\
\hline Grupo Total & 61 & 76,3 & 16 & 20,0 & 3 & 3,8 & 80 & 100,0 & \\
\hline
\end{tabular}

${ }^{*}$ ): Diferença significativa ao nível de 5,0\%.

(1): Através do teste Exato de Fisher.

(2): Através do teste Qui-Quadrado de Pearson.

Fonte: Associação entre antecedentes ginecológico-obstétricos e sintomas do climatério, 2012. 


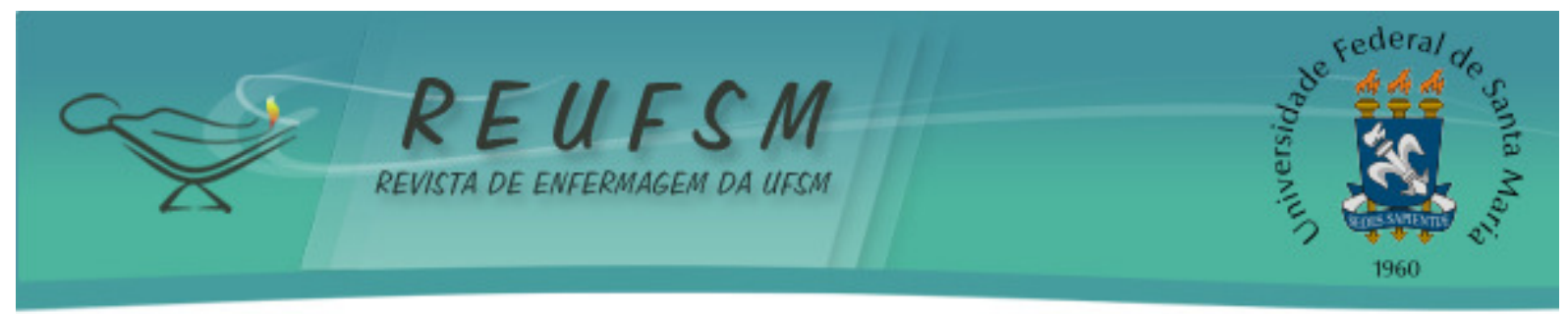

No Brasil a idade média para menarca é de 12,2 anos, ${ }^{17}$ enquanto que a atividade sexual inicia-se na faixa etária entre os 11 e 19 anos. ${ }^{18}$ Quantoa taxa de fecundidade, ${ }^{19}$ reduziu entre o grupo etário de 15 a 19 anos e, principalmente, entre o grupo de mulheres de 20 a 24 anos.

No que diz respeito às variáveis sexarca e paridade, respectivamente, o percentil dos sintomas leves foi maior entre as mulheres que tiveram a primeira relação sexual na faixa etária de 26 a 38 anos $(86,8 \%$ ), já os sintomas classificados como moderados foram mais evidenciados nas pesquisadas que tiveram sua primeira relação sexual ainda na adolescência, entre os 13 a 15 anos (37,0\%). Quanto a paridade, dos sintomas de grau leve o maior percentual foi entre as mulheres sem histórico de paridade $(77,8 \%)$, seguido das que tiveram de três ou mais partos $(76,9 \%)$, enquanto que dentre os sintomas de grau moderado a maioria foi entre as mulheres sem histórico de paridade $(22,2 \%)$, seguido das que tiveram de um a dois partos $(19,9 \%)$.

Segundo Hobeika et $\mathrm{al}^{20}$, a paridade e a menarca estão mais associados ao tempo de início da menopausa do que aos sintomas, pois mulheres nulíparas ou meninas que menstruam muito cedo tendem a entrar na menopausa mais precocemente que as demais, isto porque enquanto a menina não menstrua ou no período de gestação, a mulher poupa a liberação de óvulos. Diferentemente ocorre com as meninas que menstruam mais tarde e com as mulheres multíparas. ${ }^{20}$ Referente à primeira relação sexual, não foram encontrados achados na literatura que a correlacionasse com os sintomas do climatério.

No presente estudo foi observado que as mulheres que tinham fluxo menstrual, nenhuma apresentava sintomas intensos. Por sua vez, os sintomas de grau leve foram mais evidenciados em mulheres que ainda não tinham cessado o fluxo menstrual e que tinha fluxo regular $(88,2 \%)$, isto pode ser explicado porque um pouco menos da metade das mulheres $(47,7 \%)$ se encontravam na faixa etária de 40 a 49 anos (perimenopausa), período este que antecede a menopausa com irregularidade dos ciclos menstruais até a cessação total ou simplesmente pelo fato que nem sempre toda mulher apresenta a síndrome do climatério. ${ }^{1-2}$

Quanto ao fluxo, os sintomas de grau moderado foram mais referidos entre as mulheres que apresentavam fluxo irregular. Pode-se dizer que a síndrome do climatério tem seu início caracterizado pela irregularidade menstrual, fator que pode levar a variação de sintomas moderados a elevados. ${ }^{21}$

Das 47 mulheres que foram submetidas à histerectomia parcial 59,6\% apresentou sintomas de grau leve, o que corrobora com a literatura, visto que na histerectomia parcial ocorre a retirada do útero, mas, preservam-se os ovários, neste caso a mulher não entra em menopausa precoce e mantém o período do climatério conforme outras mulheres que não fizeram a cirurgia. ${ }^{22}$ Já nas mulheres que tinham realizado a histerectomia total, foram mais referidos os sintomas de grau moderado a intenso.

Os sintomas moderados e intensos foram mais evidenciados nas mulheres que tinham realizado a ooforectomia. A menopausa artificial induzida por ooforectomia bilateral também pode trazer sintomas dos quais são mais frequentes e de maior intensidade. ${ }^{1}$

No presente estudo a média de idade da menopausa foi de foi de 51 anos. No Brasil, a idade para a menopausa de causa natural varia entre 48 e 50 anos. ${ }^{1}$ Nos Estados Unidos a média também assume esta mesma idade de 51 anos, embora estudo norteamericano indique que a tendência para redução da faixa etária da menopausa em mulheres americanas seja maior. ${ }^{10}$ Desta forma, observa-se que a idade da menopausa, assim como seus sintomas variam de acordo com cultura, raça, fatores genéticos, uso de medicamentos, mudanças e atividades físicas, alimentação, além da história de vida pessoal e familiar, ambiente e suas próprias particularidades. 1,3,20,23 $^{2}$

Das 133 mulheres em menopausa, 80 entraram na menopausa natural e dentre elas, as que haviam cessado a menstruação há cinco anos ou mais, a maioria referiu 


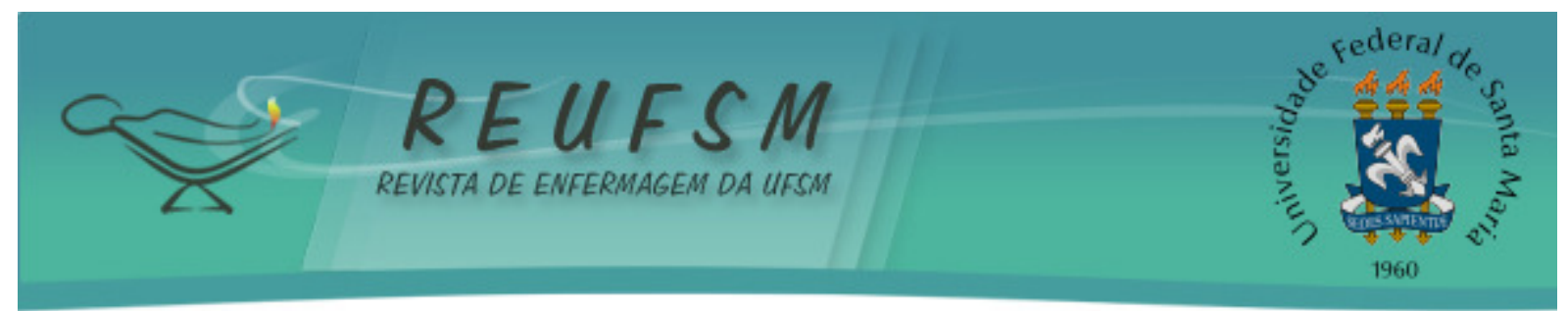

sintomatologia leve, o que entra em discordância com a literatura visto que, há relatos de maior incidência de sintomas moderado e severos no período da pós menopausa. ${ }^{19-20}$

Entretanto, alguns estudos comprovam que a prática regular de atividade física contribui para a obtenção de uma boa qualidade de vida associando-se a sintomas climatéricos menos intensos, ${ }^{23}$ este fator pode ter influenciado em nosso estudo, visto que, a população estudada consta de mulheres fisicamente ativas.

\section{CONCLUSÃO}

A associação da escala IMBK com as variáveis referentes às condições ginecológicas e obstétricas permitiu identificar que as manifestações de grau leve foram mais apontadas pelas mulheres que ainda tinham fluxo menstrual regular.

Verificou-se que os sintomas leves e moderados aumentaram com o avançar da idade, sendo os sintomas intensos menos evidenciados entre as mulheres acima dos 50 anos.

Neste estudo, dentre as 260 mulheres pesquisadas mais da metade relatou manifestações leves e apenas a minoria apresentou sintomatologia intensa, entretanto, todas eram fisicamente ativas, fator que pode ter influenciado na diminuição dos sintomas.

É de suma importância que o profissional de saúde esteja atento às manifestações clínicas presentes no climatério e os fatores que podem influenciá-las, dessa maneira poderá estabelecer um acompanhamento sistemático que objetive a promoção e a prevenção em saúde.

Assim, conclui-se que o histórico ginecológico-obstétrico pode ter relação com os possíveis sintomas do climatério, entretanto, são necessárias mais pesquisas referentes à temática abordada.

\section{REFERÊNCIAS}

1. Ministério da Saúde (BR). Secretaria de Atenção à Saúde. Departamento de Ações Programáticas Estratégicas. Manual de atenção à mulher no climatério/ menopausa. Brasília (DF): Ministério da Saúde; 2008. (Série A. Normas e Manuais Técnicos) (Série Direitos Sexuais e Direitos Reprodutivos; 9).

2. International Menopause Society. Menopause terminology [Internet]. 1999 [acesso em 2013 jun 15]. Disponível em: http://www.imsociety.org/menopause_terminology.php.

3. Rampanelli A. A prática de atividade física entre mulheres frequentadoras de academia no climatério e menopausa [monografia]. Novo Hamburgo (RS): Universidade Feevale; 2010. 58 p.

4. Pedro AO, Pinto Neto AM, Paiva LHSC, Osis MJ, Hardy E. Idade de ocorrência da menopausa natural em mulheres brasileiras: resultados de um inquérito populacional domiciliar. Cad Saúde Pública [Internet]. 2003 [acesso em 2013 jun 18];19(1):17-25. Disponível em: http://www.scielo.br/pdf/csp/v19n1/14901.pdf.

5. Pereira QLC, Silva CBDCA, Siqueira HCH. Processo de viver de mulheres climatérias usuárias do sistema único de saúde. Ciênc Cuid Saúde [Internet]. 2008 [acesso em 2013 ago 10];7(2):224-33.

Disponível

em: http://www.periodicos.uem.br/ojs/index.php/CiencCuidSaude/article/view/5006/3245.

6. Silva LD, Beck CLC, Dissen CM, Tavares JP, Budó MLD, Silva HS. O enfermeiro e a educação em saúde: um estudo bibliográfico. Rev Enferm UFSM [Internet]. 2012 maio/ago 


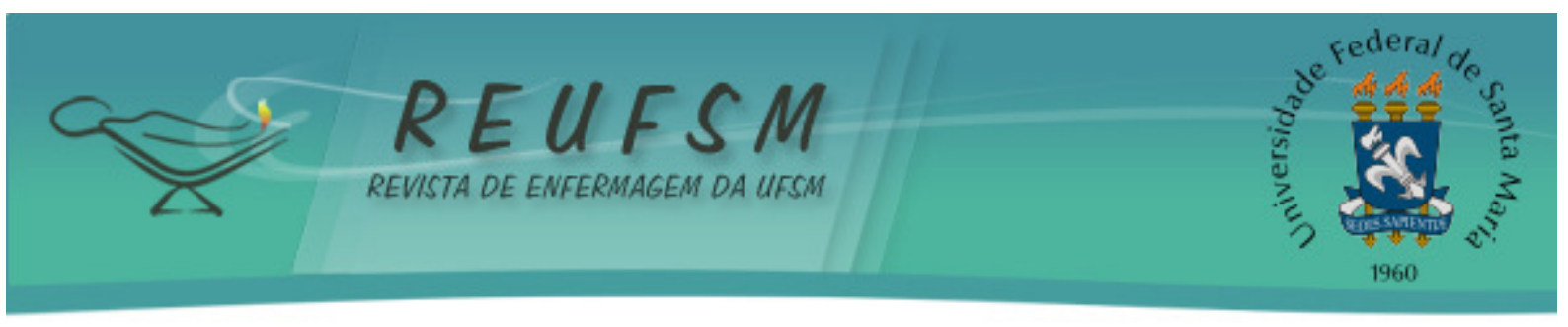

[acesso em 2013 set 10];2(2):412-9. Disponível em: http://cascavel.ufsm.br/revistas/ojs2.2.2/index.php/reufsm/article/view/2676/3769.

7. Hallal PC, Carvalho YM, Tassitano RM, Tenório MCM, Warschauer M, Reis RS, et al. Avaliação quali-quantitativa do programa academia da cidade, Recife (PE): concepções dos professores. Rev Bras Ativ Fís Saúde [Internet]. 2009 [acesso em 2013 ago 6];14(1):9-13. Disponível em: http://www.sbafs.org.br/_artigos/87.pdf.

8. Díaz-Yamal I, Munévar-Veja L. Fitoestrógenos: revisión de tema. Rev Colomb Obstet Ginecol [Internet]. 2009 [acesso em 2013 jun 15];60(3):274-80. Disponível em: http://www.fecolsog.org/userfiles/file/revista/Revista_Vol60No3_Julio_Septiembre_20 09/v60n3a08.pdf.

9. Sousa RL, Sousa ESS, Silva JCB, Filizola RG. Fidedignidade do teste-reteste na aplicação do índice menopausal de Blatt e Kupperman. Rev Bras Ginecol Obstet [Internet]. 2000 [acesso em 2013 jun 15];22(8):481-7. Disponível em: http: / /www.scielo.br/pdf/rbgo/v22n8/12063.

10. Ministério da Saúde (BR). Conselho Nacional de Saúde. Resolução no 196, de 10 de outubro 1996. Aprova as diretrizes e normas regulamentadoras de pesquisas envolvendo seres humanos. Brasília (DF): Ministério da Saúde; 1996.

11. Martins MAD, Nahas EAP, Nahas-Neto J, Uemura G, Buttros DAB, Traiman P. Qualidade de vida em mulheres na pós-menopausa, usuárias e não usuárias de terapia hormonal. Rev Bras Ginecol Obstet [Internet]. 2009 [acesso em 2013 jul 12];31(4):196-202. Disponível em: http://www.scielo.br/pdf/rbgo/v31n4/07.pdf.

12. Tairova OS, Lorenzi DRS. Influência do exercício físico na qualidade de vida de mulheres na pós-menopausa: um estudo caso-controle. Rev Bras Geriatr Gerontol [Internet]. 2011 [acesso em 2013 jul 24];14(1):135-45. Disponível em: http://revista.unati.uerj.br/pdf/rbgg/v14n1/v14n1a14.pdf.

13. Negrão RC, Moccellin AS. Importância de atividades em grupo para a qualidade de vida de mulheres pós-menopausa. Rev Bras Promoç Saúde. [Internet]. 2011 [acesso em 2013 jul 12];24(4):376-83. Disponível em: http: / / ojs.unifor.br/index.php/RBPS/article/download/2096/2389.

14. Polonini HC, Raposo NRB, Brandão MAF. A terapia de reposição hormonal e a saúde da mulher no climatério: riscos e benefícios. Rev APS [Internet]. 2011 [acesso em 2013 jun 15];14(3):354-61. Disponível em: http://www.aps.ufjf.br/index.php/aps/article/view/1129/514.

15. Kantoviski ALL, Vargens OMC. O cuidado à mulher que vivencia a menopausa sob a perspectiva da desmedicalização. Rev Eletrônica Enferm [Internet]. 2010 [acesso em 2013 ago 21];12(3):567-70. Disponível em: http://www.fen.ufg.br/fen_revista/v12/n3/v12n3a22.htm.

16. Daley AJ, Stokes-Lampard H, MacArthur C. Exercise to reduce vasomotor and othermenopausal symptoms: a review. Maturitas [Internet]. 2009 [cited 2013 Jul 6];63(3):176-80. Available from: http://www.updatesoftware.com/pdf/CD006108.pdf. 
17. Roman EP, Ribeiro RR, Guerra Junior G, Barros Filho AA. Antropometria, maturação sexual e idade da menarca de acordo com o nível socioeconômico de meninas escolares de cascavel (PR). Rev Assoc Med Bras [Internet]. 2009 [acesso em 2013 out 28];55(3):317-21. Disponível em: http: //www.scielo.br/pdf/ramb/v55n3/v55n3a26.pdf.

18. Peixoto CR, Lima TM, Costa CC, Freitas LV, Oliveira AS, Damasceno AKC. Perfil das gestantes atendidas no serviço de pré-natal das unidades básicas de saúde de Forteleza-CE. REME Rev Min Enferm [Internet]. 2012 [acesso em 2013 out 28];16(2):171-7. Disponível em: http://www.enf.ufmg.br/site_novo/modules/mastop_publish/files/files_501bf3211a106.pdf.

19. Instituto Brasileiro de Geografia e Estatística. Censo 2009 [Internet]. Rio de Janeiro; 2009 [acesso em 2013 ago 18]. Disponível em: http://www.ibge.com.br.

20. Hobeika JD, Pinto Neto AM, Paiva LHSC, Pedro AO, Martinez EZ. Histerectomia simples realizada no menacme e a densidade mineral óssea da mulher. Cad Saúde Pública [Internet]. 2002 [acesso em 2013 ago 18];18(6):1705-12. Disponível em: http://www.scielosp.org/pdf/csp/v18n6/13267.pdf.

21. Polisseni AF, Araújo DAC, Polisseni F, Mourão Junior CA, Polisseni J, Fernandes ES, et al. Depressão e ansiedade em mulheres climatéricas: fatores associados. Rev Bras Ginecol Obstet [Internet]. 2009 [acesso em 2013 ago 21];31(1):28-34. Disponível em: http://www.scielo.br/pdf/rbgo/v31n3/a03v31n3.pdf.

22. Silva CMC, Santos IMM, Vargens OMC. A repercussão da histerectomia na vida de mulheres em idade reprodutiva. Esc Anna Nery Rev Enferm [Internet]. 2010 [acesso em 2013 ago 23];14(1):76-82. Disponível em: http: / /www.scielo.br/pdf/ean/v14n1/v14n1a12.pdf.

23. Carvalho ED, Valadares ALR, Costa-Paiva LH, Pedro AO, Morais SS, Pinto-Neto AM. Atividade física e qualidade de vida em mulheres com 60 anos ou mais: fatores associados. Rev Bras Ginecol Obstet [Internet]. 2010 [acesso em 2013 ago 23];32(9):433-40. Disponível http: //www.scielo.br/pdf/rbgo/v32n9/v32n9a04.pdf.

Data de recebimento: $31 / 08 / 2013$

Data de aceite: $14 / 11 / 2013$

Contato com autor responsável: Estela Rodrigues Paiva Alves

Endereço: Edvaldo Bezerra Cavalcanti Pinho, 320. Apto. 302. Bairro do Cabo Branco. CEP: 58045-270. João Pessoa, Paraíba (PB), Brasil.

E-mail: rodrigues.estela@gmail.com 